\title{
Efectos de un taller informativo sobre la conservación de recursos hídricos en la optimización del servicio público residencial
}

\author{
Cruz García Lirios \\ Departamento de Trabajo Social, Universidad Autónoma del Estado de México. Estado \\ de México.México.cgarciali@uaemex.mx \\ ORCID: https://orcid.org/0000-0003-9364-6796
}

Javier Carreón Guillén

Escuela Nacional de Trabajo Social, Universidad Nacional Autónoma de México. Ciudad de México. México.javierg@unam.mx

ORCID: https://orcid.org/0000-0002-8915-0958

José Marcos Bustos Aguayo

Departamento de Psicología, Universidad Nacional Autónoma de México. Ciudad de

México. México. marcos.bustos@unam.mx

ORCID: https://orcid.org/0000-0002-3423-596X

Margarita Juárez Nájera

Departamento de Energía, Universidad Autónoma Metropolitana. Ciudad de México.

México.mjn@azc.uam.mx

ORCID: https://orcid.org/0000-0003-3065-9335

[Recibido: 8 julio 2020. Revisado: 13 julio 2020. Aceptado: 17 agosto 2020]

\begin{abstract}
Resumen: Los programas de educación ambiental al asumir una formación intensiva de habilidades de ahorro como el eje de solución ante la escasez de los recursos se han centrado en la optimización de los servicios públicos. En tal sentido, el objetivo del presente trabajo ha sido observar los efectos de un taller informativo en el que se promovió la prevención y la reparación de fugas, así como el ahorro del agua a través de la dosificación y la reutilización. Se realizó un estudio cuasi experimental con 316 sujetos intactos seleccionados intencionalmente. Los resultados muestran diferencias significativas antes y después del taller informativo. Es el caso del indicador de captación pluvial que en un inicio se consideró el indicador reflejante del factor, pero que luego del taller redujo su valía. En contraste, el mantenimiento de instalaciones hidráulicas develó una consciencia doméstica de conservación del patrimonio, aunque se recomienda la segmentación del taller informativo para corroborar relaciones latentes entre optimización hídrica y conservación del patrimonio.
\end{abstract}

Palabras clave: Comportamiento, vulnerabilidad, inserción, educación ambiental.

Effects of an information workshop on the conservation of water resources in the optimization of the residential public service

Abstract: Environmental education programs, assuming an intensive training of skills as the axis of solution in the face of scarce resources, have focused on the optimization of public services. In this sense, the objective of this work has been to observe the effects of an informative workshop in which the prevention and repair of leaks was promoted, as well as saving water through dosing and reuse. A quasi-experimental study was conducted with 316 intact subjects selected intentionally. The results show significant differences before and after the informative workshop. This is the case of the indicator of pluvial catchment that in a beginning was considered the reflective indicator of the factor, but that after the workshop it reduced its value. In contrast, the maintenance of hydraulic facilities revealed a domestic awareness of heritage conservation, although the segmentation of the information workshop 
is recommended to corroborate latent relationships between water optimization and heritage conservation.

Keywords: Behavior, vulnerability, insertion, environmental education.

Para citar este artículo: García, C., Carreón, J., Bustos, J. M. y Juárez, M. (2020). Efectos de un taller formativo sobre la conservción de recursos hídricos en la optimización del servicio público residencial. Revista de Educación Ambiental y Sostenibilidad 2(2), $2101 . \quad$ doi: 10.25267/Rev_educ_ambient_sostenibilidad.2020.v2.i2.2101

\section{Introducción}

En la agenda internacional prevalecen dos objetivos complementarios. Los Objetivos del Desarrollo el Milenio (ODM) y los Objetivos de Desarrollo Sostenible (ODS). En materia de recursos y servicios hídricos, los ODM aluden a generalidades relativas a exigencias vagas en planes nacionales respecto al saneamiento. Son las ODS las que delimitan ejes de acción vinculantes a programas locales como acceso universal y equitativo (Sanahuja y Tezanos, 2017). Las diferencias entre ODM y ODS son evidentes por la construcción de una agenda a partir de la especificación operacional de conceptos y su prueba empírica de los mismos.

Destaca el sexto ODS relativo al agua limpia y saneamiento ambiental. Se trata de un balance entre la disponibilidad de los recursos hídricos y la gestión de la salubridad mínima. Alude a los efectos de la contaminación de los cuerpos de agua sobre la hidratación y cultivo de productos agrícolas. Puntualmente, se propone al desarrollo tecnológico en la desalinización, reutilización y reciclaje como una medida de sostenibilidad (Gamboa, 2015). La generalidad de estas propuestas supone una agenda de especificación de temas para su discusión.

A pesar de que la disponibilidad de los recursos hídricos es cada vez menor y el acceso a un servicio de calidad es limitado, la agenda enuncia los problemas de contaminación y sobreexplotación de los cuerpos de agua, pero los problemas de sequía se intensifican aún en zonas desarrolladas como California (Estados Unidos) donde se han abandonado las plantaciones por escasez o desabastecimiento (Colmenarejo, 2016). Es decir, los ODS pueden avanzar en cuanto a su especificación a partir de los escenarios regionales o situaciones locales.

Precisamente, la Educación para la Sustentabilidad, entendida como un proceso de cambio de actitudes y capacidades a partir de la disponibilidad de recursos, el acceso equitativo y la tecnología de desalinización, tratamiento y reutilización supone un instrumento de diagnóstico e intervención para el alcance del ODS-6. Esto es así porque la promoción de relaciones equitativas, la prevención de riesgos e intervención a través de la tecnología ante la escasez, desabastecimiento, insalubridad y carestía son metas asequibles para el ODS-6.

Por consiguiente, el objetivo del presente trabajo es contribuir con evidencia empírica a los indicadores del ODS-6: escasez, sequia, agotamiento, saneamiento, potabilización, calidad e inundaciones (Gain, Giupponi y Wada, 2016). Para tal propósito, se evalúan los efectos de un programa informativo, considerando estos indicadores ODS como parte de un constructo que la literatura identifica como comportamiento para la sustentabilidad la cual define como una acción deliberada, planificada y sistemática de optimización de recursos e innovación de procesos a 
favor de la conservación de especies animales y vegetales para las futuras generaciones (Valizadeh, Bijani y Hayati, 2019).

¿Cuáles son los efectos de un programa informativo centrado en los indicadores hídricos de los ODS-6, considerando la Escala de Orientación a la Sustentabilidad y su estructura factorial de ocho dimensiones concomitantes entre sí y reflejantes en un factor común en estudiantes de una universidad pública?

Las hipótesis que guían el presente trabajo sugieren:

1) confirmación de la estructura de la Escala de Orientación a la Sustentabilidad, la cual incluye ocho dimensiones relativas a austeridad, altruismo, proambientalismo, deliberación, afinidad, indignación, autopresentación y normatividad;

2) diferencias significativas entre los indicadores del ODS-6 con respecto a sus efectos observables en la Escala de Orientación a la Sustentabilidad.

En virtud de que la discusión radica en las diferencias institucionales por área de conocimiento, el presente trabajo incluye un apartado dedicado a la Teoría Institucionalista para la sustentabilidad con la finalidad de esclarecer los niveles de intervención y los efectos de programas informativos. En seguida, se expone el modelamiento de los ejes, trayectorias y relaciones entre los indicadores como parte del comportamiento para la sustentabilidad a fin de poder explicar las diferencias entre disciplinas. Se incluye el abordaje metodológico, los resultados y la discusión con base en los presupuestos teóricos de la educación y el comportamiento para la sustentabilidad.

\section{Teoría institucionalista para la sustentabilidad}

En el presente apartado se expone la matriz teórica y conceptual relativa al ODS-6 en las Instituciones de Educación Superior (IES) para esclarecer las diferencias entre localidades e instituciones al momento de observar un aprendizaje de los indicadores del ODS-6. De esta manera, se considera que los niveles de explicación son consustanciales a las intervenciones (Little, 2014). En el caso de los programas informativos es menester tener en cuenta algunos hallazgos reportados en la literatura seleccionada y consultada.

En virtud de que la educación para la sustentabilidad (ES) refiere a una consciencia de los efectos del cambio climático sobre la salud pública ambiental, en el marco de las IES alude a una formación integral (Kumar, 2014). Es decir, la ES como un ámbito en el que el ODS-6 se transfiere devela un sistema de gestión, producción y transferencia del conocimiento. En otras palabras, escasez, sequia, agotamiento, saneamiento, potabilización, calidad e inundaciones son datos asequibles, asimilables y traducibles en acciones de optimización en el consumo institucional de agua.

Es así como el ODS-6 y la ES se entrelazan en el plano institucional de las universidades públicas al establecer programas de conciencia, ahorro y reutilización institucional con el propósito de reducir la huella hídrica (Milana, Rasmussen y Holford, 2014). Se trata de un proceso deliberado, planificado y sistemático en el que el ODS-6 es traducido a la misión y visión institucional. Incluso, la evaluación, acreditación y certificación de la calidad de procesos y productos ya incluye este rubro de sustentabilidad hídrica. 
Sin embargo, los estudios parecen centrar su interés en el indicador de la escasez más que en los otros indicadores del ODS-6. Es decir, a nivel institucional, el ODS-6 está delimitada a un indicador preponderante que alude a la optimización de los recursos hídricos a través del servicio de agua potable, reutilización y tratamiento.

A ese tenor, los indicadores del ODS-6 han sido traducidos desde una conciencia y acción reactiva ante la escasez a una anticipación de desabastecimiento. De este modo, la difusión del saneamiento determinó la salud comunitaria, una correlación positiva entre las medidas preventivas con respecto a la optimización de recursos y el aprovechamiento escolar (Noga y Wolbring, 2012).

Los indicadores del ODS-6 aluden a una conciencia personal y colectiva de escasez de los recursos, pero reversible a partir de la formación de optimizadores de los recursos, cuidado y ahorro del agua. En ese sentido es que la evaluación de los efectos de un taller informativo sobre la escasez permitirá especificar su análisis institucional.

\section{Modelamiento de los indicadores ODS-6 en una IES}

En esta sección se exponen los ejes, trayectorias y relaciones entre los indicadores del ODS-6 con respecto a las IES y su comportamiento para la sustentabilidad. Se plantean escenarios de escasez, desabastecimiento, insalubridad y carestía ante los cuales las IES reaccionarían, pero con matices de acuerdo con las capacidades de las áreas de conocimiento como es el caso de las ciencias de la salud y las ciencias económicas administrativas.

Por consiguiente, un modelamiento es un mapeo de las variables indicativas de un comportamiento a partir de criterios de inclusión como el consenso de la literatura en cuanto al ODS-6 y su observación en las IES.

Si las IES filtran la información a partir de sus necesidades, capacidades, expectativas y recursos, entonces se traducen en escasez, desabastecimiento, insalubridad, reciclaje, saneamiento, reutilización y carestía. Estos indicadores institucionales pueden ser observados en las divisiones de salud y economía, aunque con sus acentuaciones. Se espera que en las ciencias biológicas y de la salud los indicadores del ODS-6 sean más conocidos que en las ciencias económico-administrativas.

De este modo, en las ciencias biológicas y de la salud prevalecen encuadres de verificabilidad. Es decir, la difusión de los indicadores del ODS-6 propician una disposición a comprobar sus contenidos con diferentes fuentes. De hecho, un comparativo entre diversas fuentes observando el mismo fenómeno genera la veracidad de un dato.

En contraste, las ciencias económico-administrativas, distantes a los indicadores del ODS-6, desarrollan una lógica de verosimilitud que consiste en aceptar una difusión informativa por su estructura de contenidos o secuencias de datos más que por el consenso de diversas fuentes. Es así como una nota relativa a la escasez de agua es verosímil si los receptores han sido formados con ese principio de desabastecimiento de agua en la institución y por consiguiente su cuidado. 
Ambos fenómenos, verificabilidad y verosimilitud, coexisten en las instituciones que adoptaron los indicadores del ODS-6 y los difundieron como escasez, desabastecimiento, insalubridad, saneamiento, carestía, reciclaje y reutilización.

La IES de estudio está ubicada en el centro de México, región de mediano desarrollo humano, ingreso per cápita inferior a la media de 7500 pesos mensuales, con una tasa de natalidad superior a la media nacional del 7\% anual, densidad de 3 personas por 20 metros cuadrados de construcción, así como de baja disponibilidad hídrica con sistema de tandeo de dos o tres veces por semana, durante un promedio de tres horas. Cerca del $20 \%$ del ingreso familiar es invertido en la compra de agua embotellada, aunque las enfermedades hidro-transmitidas ocasionan la muerte de infantes más que de adultos. La IES solo cubre unas cuantas disciplinas que obedecen a las necesidades de desarrollo de la región, así como la demanda laboral proyectada.

\section{Método}

\section{Diseño}

La medición de los indicadores del ODS-6 en la IES siguió un estudio cuasi experimental con una variante transversal de comparación inter-sujetos y una asignación no aleatoria (Ato, López y Benavente, 2013).

\section{Primera fase}

Se realizó una selección no probabilística e intencional con 316 estudiantes ( $\mathrm{M}=23.2$ con $\mathrm{DE}=2.1$ de edad y $\mathrm{M}=8983.24$ con $\mathrm{DE}=435.46$ de ingreso mensual) de la UAEM de las licenciaturas de Economía, Relaciones Públicas, Comunicación Humana y Psicología. Se llevó a cabo la fiabilidad y la validez de un instrumento diseñado a partir de la Escala de Orientación hacia la Sustentabilidad de Corral, Tapia, Fraijo, Mireles y Márquez (2008). Incluye ocho dimensiones relativas:

1) austeridad con seis acciones que limita el consumo y el desperdicio de recursos la cual alcanzó un coeficiente alfa de consistencia interna de 0.60 en la versión original y de 0.65 en la versión final del presente estudio,

2) altruismo con diez aseveraciones sobre ayuda desinteresada y alfa de 0.70 en la versión original y 0.68 en la versión final,

3) proambientalismo con 11 reportes de comportamiento de ahorro, reutilización y reciclaje con alfa de 0.74 versión final y 0.70 en versión final,

4) deliberación en la protección de especies con 10 ítems y alfa de 0.78 en la versión original y 0.70 en la versión final,

5) afinidad por la diversidad con 4 enunciados y alfa de 0.68 para versión original y 0.73 para versión final,

6) indignación por el deterioro ambiental con siete ítems y alfa de 0.79 en versión original y 0.66 en versión final de siete reactivos,

7) autopresentación con ocho ítems que miden la valoración de acciones con alfa de 0.74 para versión original y alfa de 0.66 en versión final,

8) normatividad con cinco reactivos y alfa de 0.90 para versión original y 0.86 para versión final. 
La Validez se estableció con pesos factoriales entre 0.342 y 0.561 entre los indicadores y las subescalas, previa adecuación y esfericidad $\left[\mathrm{X}^{2}=13.24\right.$ (23 g.l.) $\left.\mathrm{p}<0.05 ; \mathrm{KO}=0.680\right]$.

\section{Segunda fase}

Se realizó una selección no probabilística e intencional con 100 estudiantes $(M=21.4$ con $\mathrm{DE}=3.21$ de edad y $\mathrm{M}=7345.23$ pesos con $\mathrm{DE}=541.36$ pesos de ingreso mensual) en las mismas licenciaturas de Economía, Relaciones Públicas, Comunicación Humana y Psicología. Se implementó el Taller de Educación para la Sustentabilidad con la finalidad de diseminar los indicadores del ODS-6 en la comunidad universitaria, fomentar las prácticas en docentes y administrativos. Durante el periodo que va de enero de 2018 a diciembre de 2019 en siete sesiones se promovieron los indicadores del ODS-6 traducidos en dimensiones de escasez, desabastecimiento, insalubridad, carestía, reutilización reciclaje y saneamiento. Cada sesión duró dos horas con una secuencia pedagógica introductoria a los indicadores del ODS-6, seguida de una trasferencia de conocimiento en juegos de roles y con sesión de preguntas y respuestas al finalizar. Cada participante firmó una carta de consentimiento informado en donde se les notificó que su participación no sería remunerada. Se garantizó la confidencialidad, anonimato y no afectación de los participantes $y$, se facilitaron los datos de las instituciones e investigadores responsables del proyecto.

Al finalizar el total de las sesiones se procedió a aplicar la escala basada en la orientación hacia la sustentabilidad:

1) austeridad con alfa de 0.65 y $16 \%$ de la varianza total explicada,

2) altruismo con alfa de 0.63 y $13 \%$ de la varianza total explicada,

3) conducta ecológica general con alfa de 0.68 y $10 \%$ de la varianza total explicada,

4) deliberación con alfa de 0.70 y $7 \%$ de la varianza total explicada,

5) afinidad con alfa de 0.78 y $5 \%$ de la varianza total explicada,

6) indignación con alfa de 0.69 y $3 \%$ de la varianza total explicada,

7) autopresentación con alfa de 0.62 y $2 \%$ de la varianza total explicada,

8) normatividad con alfa de 0.80 y $1 \%$ de la varianza total explicada.

\section{Procedimiento}

El instrumento se aplicó a estudiantes de la Universidad Autónoma del Estado de Morelos (UAEM) en las sedes que concentran el mayor número de estudiantes de esta universidad: Chamilpa y Cuautla.

El cuestionario se adaptó a fin de que los estudiantes lo contestaran en línea de forma voluntaria sin la presencia de un encuestador, evitando en lo posible el sesgo en sus respuestas. Los datos se agruparon en la plataforma Google Forms desde la cual fueron descargados a MS-Excel 2013 a fin de realizar su validación, así como su depuración, desechando 11 cuestionarios que presentaron inconsistencias. Posteriormente se exportaron a IBM-SPSS-AMOS v.25 donde se realizaron los análisis estadísticos correspondientes. Considerando que la UAEM tiene cerca de 50 mil estudiantes se tomó este como el universo, por lo cual el error muestral es de $5.6 \%$ con un nivel de confiabilidad de $95 \%$ de conformidad con la fórmula siguiente: 


$$
n=\frac{(p q)^{2} z^{2}}{e^{2}}
$$

Se realizó una distribución normal considerado los parámetros de curtosis univariada y multivariada, así como análisis de sesgo. La fiabilidad se estimó con el parámetro alfa de Cronbach el cual identifica el error de medición a partir de un mínimo de 250 sujetos y la comparación entre ellos (Merino, 2016). La validez se estableció con un análisis factorial confirmatorio de la estructura reportada en la Escala de Orientación a la Sustentabilidad a fin de poder contrastar la hipótesis de número de factores y relaciones tanto entre las dimensiones como con respecto a un factor de segundo orden común (Ferrando y Anguiano, 2010). Se contrastaron modelos de ecuaciones estructurales, considerando:

1) identificación o exclusión de varianzas negativas y número igual o menor de relaciones con respecto a variables observables;

2) estimación o contraste de la hipótesis de que la matriz de varianzas y covarianzas poblacional es igual a la matriz de varianzas y covarianzas del modelo teórico, considerando la máxima verosimilitud;

3) ajuste o prueba empírica de la matriz de varianzas y covarianzas muestral con respecto a la identificada en los parámetros del modelo (Manzano, 2017).

\section{Resultados}

En este apartado se exponen los hallazgos relativos a la fiabilidad y validez de la Escala de Orientación hacia la Sustentabilidad, así como las diferencias de esta una vez que se ha intervenido en una muestra de estudiantes a través de un taller informativo de los indicadores del ODS-6. Se rechazó la hipótesis nula relativa a que no existen diferencias significativas antes y después de implementado el taller informativo.

La Tabla 1 muestra las propiedades estadísticas del instrumento que midió los ocho factores del comportamiento proambiental relativo a la conservación del agua. Es posible observar que los valores de sesgo y curtosis permiten inferir la distribución normal de las respuestas al inventario, aunque el valor que determina la distribución normal es la curtosis multivariada que alcanzó un valor apenas superior al mínimo de 15.00. Estos resultados justifican los análisis de fiabilidad y validez. La distribución normal de las respuestas a los reactivos sugiere que los indicadores del ODS- 6 al estar representados por la Escala de Orientación hacia la Sustentabilidad son conocidos por los estudiantes antes del taller informativo sobre esta materia.

Tabla 1. Propiedades estadísticas del instrumento anterior al taller

\begin{tabular}{ccccc}
\hline Factor & MN & MX & S & CU \\
\hline F1 & 1.000 & 5.000 & 0.610 & -0.540 \\
\hline F2 & 1.000 & 5.000 & -0.202 & -0.837 \\
\hline F3 & 1.000 & 5.000 & 1.748 & 2.821 \\
\hline F4 & 1.000 & 5.000 & -0.389 & -0.785 \\
\hline F5 & 1.000 & 4.000 & 1.317 & 0.851 \\
\hline F6 & 1.000 & 5.000 & -0.190 & -0.589 \\
\hline F7 & 2.000 & 5.000 & -0.702 & -0.226 \\
\hline F8 & 1.000 & 5.000 & 1.423 & 0.711 \\
\hline CM & & & & 15.088
\end{tabular}

$\mathrm{R}=$ Reactivo, $\mathrm{MN}=\overline{\text { Mínimo }, \mathrm{MX}=\text { Máximo }, \mathrm{S}=\text { Sesgo, } \mathrm{CU}=\text { Curtosis Univariada }, \mathrm{CM}}=$ Curtosis Multivariada 
Una vez establecida la distribución normal de los factores del comportamiento proambiental, se procedió a estimar sus correlaciones y covarianzas con la finalidad de medir la incidencia de otros factores asociados al constructo de la conservación del agua (véase Tabla 2 y 3). Estos hallazgos develan la estructura de relaciones entre las dimensiones de la orientación hacia la sustentabilidad. Significa entonces que se trata de una estructura consistente con la teoría reportada en la literatura con respecto a los factores de la Escala de Orientación hacia la Sustentabilidad.

Tabla 2. Correlaciones entre factores

\begin{tabular}{rrrrrrrrr}
\hline & F8 & F7 & F6 & F5 & F4 & F3 & F2 & F1 \\
\hline F8 & 1.000 & & & & & & & \\
\hline F7 & 0.111 & 1.000 & & & & & & \\
\hline F6 & 0.438 & 0.072 & 1.000 & & & & & \\
\hline F5 & 0.035 & 0.311 & 0.082 & 1.000 & & & & \\
\hline F4 & 0.420 & 0.052 & 0.316 & -0.073 & 1.000 & & & \\
\hline F3 & 0.124 & 0.323 & 0.190 & 0.350 & 0.201 & 1.000 & & \\
\hline F2 & -0.046 & 0.279 & 0.082 & 0.143 & -0.086 & 0.149 & 1.000 & \\
\hline F1 & 0.354 & 0.228 & 0.434 & 0.220 & 0.165 & 0.117 & 0.041 & 1.000 \\
\hline
\end{tabular}

Condition number $=5.255$; Eigenvalues: $2.345 ; 1.606 ; 0.938 ; 0.895 ; 0.703 ; 0.560 ; 0.507 ; 0.446$

Las correlaciones muestran una prevalecía de asociaciones positivas entre los factores, aunque en algunos casos esa relación fue espuria y negativa, condición para asumir que los factores están asociados a un constructo común. Esto es así porque la orientación hacia la sustentabilidad al ser un factor común incide en las ocho dimensiones reportadas en la literatura y observadas en el presente trabajo. Por consiguiente, los indicadores del ODS-6 son identificables en esta estructura de relaciones en donde este constructo de orientación hacia la sustentabilidad explica hasta en un $57 \%$ de la varianza total.

Tabla 3. Covarianzas entre factores

\begin{tabular}{rrrrrrrrr}
\hline & F8 & F7 & F6 & F5 & F4 & F3 & F2 & F1 \\
\hline F8 & 1.039 & & & & & & & \\
\hline F7 & 0.128 & 1.273 & & & & & & \\
\hline F6 & 0.382 & 0.070 & 0.733 & & & & & \\
\hline F5 & 0.037 & 0.367 & 0.073 & 1.092 & & & & \\
\hline F4 & 0.386 & 0.053 & 0.244 & -0.069 & 0.813 & & & \\
\hline F3 & 0.132 & 0.382 & 0.170 & 0.383 & 0.190 & 1.096 & & \\
\hline F2 & -0.043 & 0.289 & 0.064 & 0.137 & -0.071 & 0.143 & 0.842 & \\
\hline F1 & 0.433 & 0.309 & 0.445 & 0.276 & 0.178 & 0.147 & 0.045 & 1.435 \\
\hline
\end{tabular}

Condition number $=6.347$; Eigenvalues: $2.555 ; 1.636 ; 1.043 ; 0.887 ; 0.715 ; 0.620 ; 0.465 ; 0.403$;

Determinant of sample covariance matrix $=0.321$

En el caso de las covarianzas, los valores muestran que otros factores podrían ser incluidos en el factor de conservación de agua, pero su valor aproximado al cero supone su convergencia en el factor y la no incidencia de otros factores. Es decir, la estructura de los indicadores del ODS-6 medida a través de la Escala de Orientación a la Sustentabilidad es homogénea. En contextos y muestras diferentes estas relaciones entre factores no varían significativamente. Por lo tanto, la observación de un constructo reflejado por ocho dimensiones puede ser observada posterior a un taller informativo. 
El modelo estructural de trayectorias reflejantes muestra que la captación pluvial es el principal indicador que refleja la conservación del agua. Es decir, la muestra encuestada parece evidenciar una propensión a la adopción de tecnología de captación pluvial más que otras acciones registradas en el inventario. Tal hallazgo, permite observar los indicadores del ODS-6 en un ámbito residencial como parte de una orientación hacia la sustentabilidad en la muestra encuestada. Es decir, los estudiantes adquieren comportamientos afines al ODS-6 en materia de austeridad.

Los parámetros de ajuste $\left[\mathrm{X}^{2}=345.24\right.$ (23 g.l.) $\mathrm{p}=0.005$; CFI $=0.990$; GFI $=0.995$; RMSEA $=0.006]$ sugieren la aceptación de la hipótesis nula relativa a las no diferencias significativas entre la especificación del modelo con respecto a la estructura de las relaciones entre el constructo y los factores. Esto es así porque la orientación hacia a sustentabilidad en el norte del país donde se generó la escala es diferente.

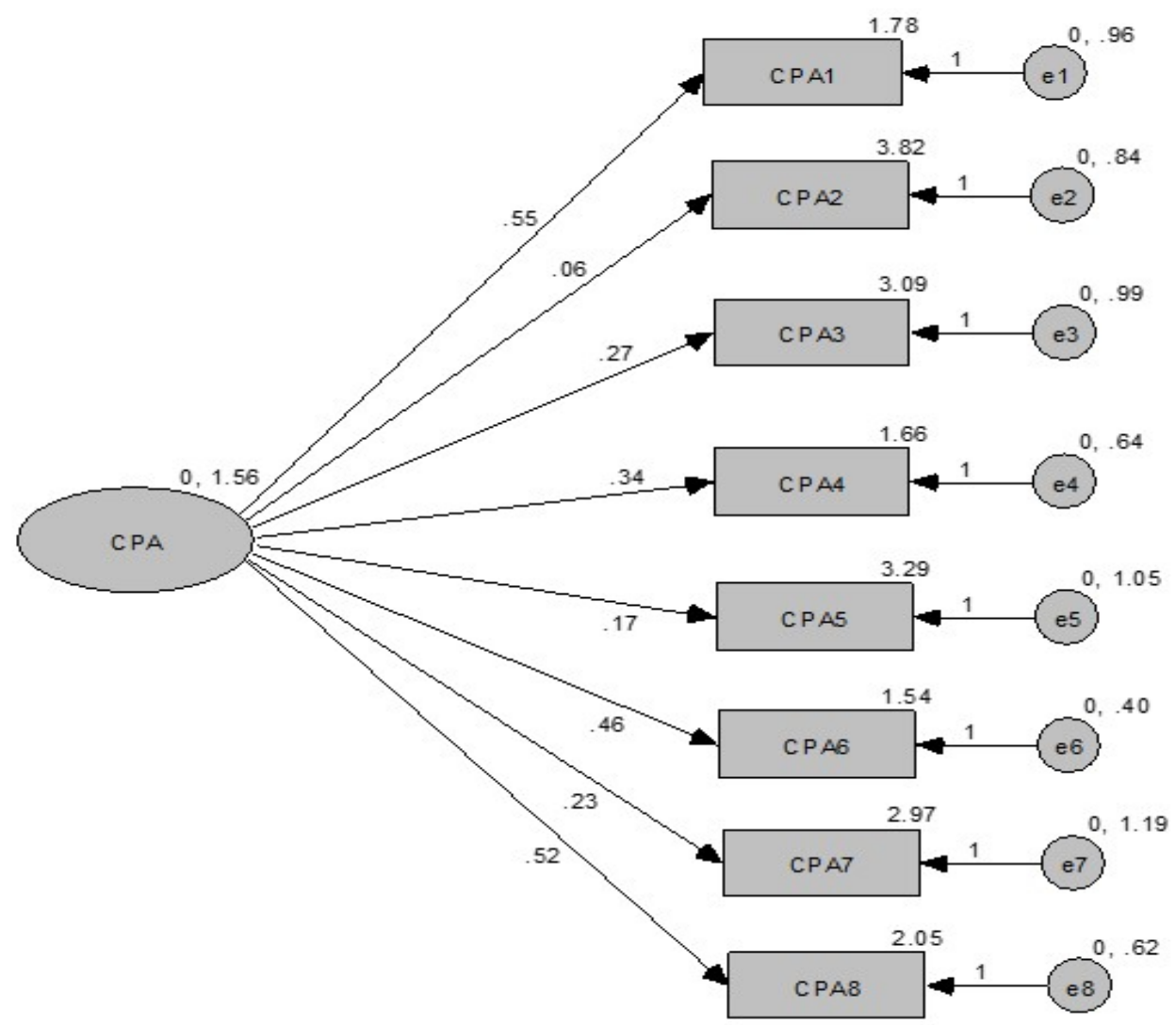

Figura 1. Modelo estructural reflejante del comportamiento proambiental

Una vez establecido el primer diagnóstico se procedió a realizar un segundo estudio luego de la intervención centrada en un taller de promoción del ahorro de agua, considerando ocho factores:

1) la captación pluvia (austeridad),

2) el almacenamiento planificado (altruismo),

3) la optimización del recurso (proambientalismo),

4) la dosificación del consumo (deliberación),

5) la reutilización del agua (afinidad), 
6) la detección de fugas (indignación),

7) la reparación de fugas (autopresentación),

8) el mantenimiento hidráulico (normatividad).

La Tabla 4 muestra las propiedades psicométricas del instrumento que se aplicó después del taller informativo sobre prevención, reparación, mantenimiento y ahorro de agua en las residencias.

Tabla 4. Propiedades estadísticas del instrumento posterior al taller

\begin{tabular}{ccccc}
\hline F & MN & MX & S & CU \\
\hline F1 & 1.000 & 5.000 & 0.317 & 0.723 \\
\hline F2 & 1.000 & 5.000 & 0.080 & 0.155 \\
\hline F3 & 1.000 & 5.000 & 1.952 & 0.182 \\
\hline F4 & 1.000 & 5.000 & 1.662 & 960 \\
\hline F5 & 1.000 & 5.000 & -1.583 & 0.584 \\
\hline F6 & 1.000 & 5.000 & 0.477 & -0.237 \\
\hline F7 & 1.000 & 5.000 & -1.799 & 0.699 \\
\hline F8 & 1.000 & 4.000 & 0.083 & 0.262 \\
\hline CM & & & & 58.030
\end{tabular}

$\mathrm{R}=$ Reactivo, $\mathrm{MN}=$ Mínimo, $\mathrm{MX}=$ Máximo, $\mathrm{S}=$ Sesgo, $\mathrm{CU}=$ Curtosis Univarable, $\mathrm{CM}=$ Curtosis Multivariable

Es posible observar que los factores mantienen una distribución normal, aunque la curtosis multivariable se disparó de 15.088 en el primer diagnóstico a 58.030 en el segundo diagnóstico (véase Tabla 4 y 5). Este fue el primer indicio de diferencias significativas antes y después del taller informativo. Tal hallazgo sugiere el perfeccionamiento de los indicadores del ODS-6, considerando la medición de algunos rasgos en la Escala de Orientación hacia la Sustentabilidad.

Tabla 5. Correlaciones entre los factores

\begin{tabular}{ccccccccc}
\hline & F8 & F7 & F6 & F5 & F4 & F3 & F2 & F1 \\
\hline F8 & 1.000 & & & & & & & \\
\hline F7 & 0.620 & 1.000 & & & & & & \\
\hline F6 & 0.628 & 0.594 & 1.000 & & & & & \\
\hline F5 & 0.429 & 0.511 & 0.630 & 1.000 & & & & \\
\hline F4 & -0.542 & -0.399 & -0.310 & -0.174 & 1.000 & & & \\
\hline F3 & 0.373 & 0.325 & 0.277 & 0.207 & -0.456 & 1.000 & & \\
\hline F2 & -0.460 & -0.393 & -0.297 & -0.079 & 0.554 & -0.358 & 1.000 & \\
\hline F1 & 0.574 & 0.456 & 0.526 & 0.310 & -0.545 & 0.336 & -0.320 & 1.000 \\
\hline
\end{tabular}

En el caso de las correlaciones entre los factores se observa un incremento de sus valores negativos lo cual sugiere la posible relación de esos factores con otro factor, pero las bajas covarianzas entre estos factores denotan que el factor de conservación sólo estaría siendo observado e inferido por estos ocho factores (véase Tabla 6). Tales resultados develan una estructura de relaciones entre variables que explican la incidencia del taller informativo en los factores de la escala. Esta consistencia de resultados antes y después del taller informativo sugiere que la muestra encuestada ya tenía asimilados los indicadores del ODS-6 y el taller vino a incrementar su orientación hacia la sustentabilidad. 
Tabla 6. Covarianzas entre factores

\begin{tabular}{ccccccccc}
\hline & F8 & F7 & F6 & F5 & F4 & F3 & F2 & F1 \\
\hline F8 & 0.598 & & & & & & & \\
\hline F7 & 0.398 & 0.690 & & & & & & \\
\hline F6 & 0.385 & 0.391 & 0.629 & & & & & \\
\hline F5 & 0.312 & 0.399 & 0.470 & 0.884 & & & & \\
\hline F4 & -0.347 & -0.275 & -0.204 & -0.136 & 0.686 & & & \\
\hline F3 & 0.265 & 0.248 & 0.202 & 0.179 & -0.348 & 0.847 & & \\
\hline F2 & -0.286 & -0.262 & -0.189 & -0.060 & 0.370 & -0.265 & 0.648 & \\
\hline F1 & 0.354 & 0.302 & 0.332 & 0.233 & -0.360 & 0.247 & -0.205 & 0.635 \\
\hline
\end{tabular}

Condition number $=16.419 ;$ Eigenvalues: $2.726 ; 0.961 ; 0.559 ; 0.414 ; 0.310 ; 0.266 ; 0.214 ; 0.166$;

Determinant of sample covariance matrix $=0.002$

Sin embargo, la diferencia más significativa en torno a los efectos del taller es posible observarla en el primer indicador que paso de ser el principal con un valor de 0.55 al cuarto lugar con 0.47 evidenciando que la captación pluvial ya no reflejaba la conservación del agua.

En contraste, el último indicador relativo al mantenimiento de las instalaciones hidráulicas ubicado en segundo lugar de importancia con 0.52 pasó a ser el primer indicador reflejante con 0.57 luego de haberse efectuado el taller.

Ambos hallazgos, la reducción de la captación pluvial y el incremento del mantenimiento a las instalaciones hidráulicas sugieren que los indicadores del ODS-6 reflejan una evolución social en materia de cuidado de los recursos que puede ser intensificada por el taller informativo.



Figura 2. Modelo estructural reflejante posterior al taller 
Por último, los parámetros de ajuste $\left[\mathrm{X}^{2}=345.25\right.$ (34 g.l.) $\mathrm{p}=0.005$; GFI $=0.990$; $\mathrm{CFI}=$ 0.995 ; RMSEA $=0.008$ ] sugieren el rechazo de la hipótesis nula relativa a las no diferencias antes y después del taller informativo.

Por lo tanto, el taller informativo incidió positivamente en el factor normativo e institucional de reparación de instalaciones hidráulicas, aunque negativamente en la austeridad observable en la captación pluvial.

\section{Discusión}

El presente trabajo ha demostrado diferencias significativas antes y después de implementado un taller informativo sobre los recursos y servicios hídricos, considerando ocho dimensiones orientadas hacia la sustentabilidad, aunque el diseño de la investigación limita estos hallazgos a la muestra de investigación, sugiriendo la extensión del trabajo a otros escenarios y muestras.

En virtud de los parámetros de contraste encontrados se rechazaron las hipótesis relativas a:

1) la confirmación de la estructura factorial con orientación hacia la sustentabilidad, aunque prevalecen las ocho dimensiones alusivas a austeridad, altruismo, proambientalismo, deliberación, afinidad, indignación, autopresentación y normatividad;

2) diferencias entre los indicadores del ODS-6 reportados en la literatura con respecto a los observados en el presente trabajo.

Yazici (2020) implementó un curso de hidrografía para observar la concientización de estudiantes la cual fue concomitante con sus creencias relativas a la efectividad de sus acciones sobre el impacto del cambio climático y sus efectos en la distribución de los sistemas terrestres y la biodiversidad, así como el reciclaje, destacando la atribución al Estado como rector de las políticas contra el cambio climático y sus efectos.

En el presente trabajo se ha observado que las problemáticas hídricas, medidas mediante ocho indicadores, se orientan hacia un constructo simbólico de sustentabilidad, pero la configuración de su estructura factorial es diferente con respecto al escenario y la muestra del estudio original. Esto es así porque los indicadores del ODS-6 parecen ser asimilados indistintamente en las regiones y localidades, considerando su grado de orientación hacia la sustentabilidad.

Maletic, Maletic, Dahlgaard, Dahlgaard-Park y Gomiscek, (2016) demostraron que la calidad y la innovación orientadas hacia la sustentabilidad propician beneficios, utilidades y ganancias traducidas en mejoras económicas y financieras para las organizaciones que adoptan estas responsabilidades sociales.

En el presente estudio se ha evidenciado que, si bien ocho dimensiones se orientan hacia la sustentabilidad y configuran una estructura factorial acorde a la reportada en la literatura, prevalecen diferencias en cuanto a las relaciones entre las variables. Tales cuestiones reflejan las diferencias entre localidades y su representación del ODS-6. Este mismo detalle puede ser observado en las propuestas de orientación hacia la sustentabilidad tanto en organizaciones como en instituciones. Es decir, los lineamientos globales de los indicadores del ODS-6 se diseminan conforme a los recursos y servicios hídricos disponibles, pero también en función de las capacidades; 
habilidades, conocimientos y saberes de grupos o sectores, inducidos o no por talleres informativos para orientar sus conductas hacia la conservación del agua.

La teoría institucionalista orientada hacia la sustentabilidad advierte la asimilación de los indicadores del ODS-6 sobre los integrantes de las IES como los docentes y estudiantes. A través de la misión y visión institucional la teoría señala que se diseminan los indicadores del ODS-6 identificando problemáticas y protocolos dirigidos a reducir el impacto o la huella hídrica, así como a maximizar la conservación de los recursos y servicios hídricos.

En el presente trabajo, la observación de esa orientación institucional hacia la sustentabilidad consiste en ocho dimensiones las cuales se representan asimétricamente antes y después de un taller, sugiriendo que esas diferencias se extienden a nivel local o regional.

En cuanto a la especificación del modelo para el estudio de la orientación hacia la sustentabilidad donde se suponen diferencias entre las partes involucradas antes y después de un taller informativo, el presente trabajo ha demostrado que tales asimetrías se deben a la exposición de ocho factores, pero el diseño de la investigación limita este hallazgo a la muestra, sugiriendo un rediseño experimental para el control y manipulación de variables que permitan develar el efecto del taller informativo.

\section{Conclusión}

El objetivo del presente trabajo ha sido establecer los efectos de un programa informativo sobre la importancia de prevenir y reparar las fugas residenciales, así como la conservación del agua a partir del ahorro, considerando una conciencia ecológica.

Sin embargo, el tipo de estudio cuasi experimental limita los resultados a la muestra de participantes, aunque resulta de especial importancia el contraste de modelos en los que se observa al mantenimiento de las instalaciones hidráulicas como el principal indicador de la conservación de agua.

El tipo de estudio con sujetos intactos limita los resultados a la muestra de participantes, aunque el indicador que finalmente reflejó la conservación del agua parece mostrar una propensión al cuidado de las instalaciones del hogar más que la conciencia de ahorrar agua para las futuras generaciones.

Ello implicaría el diseño de un taller más efectivo ya que, la promoción de la prevención y reparación de fugas parece estar más orientada a la conservación del patrimonio. En consecuencia, la optimización de los recursos estaría vinculada a la conciencia de preservar el agua para las futuras generaciones.

Es menester segmentar el taller y orientarlo de acuerdo con estos hallazgos con la finalidad de innovar los procesos de mantenimiento de las instalaciones hidráulicas a partir de la conservación del patrimonio residencial, a la vez, promover la optimización de los recursos hídricos con base en una conciencia de ahorro para las futuras generaciones.

Nuevas interrogantes emergen del presente trabajo; ¿por qué los ODS-6 se diseminan simétricamente entre escenarios y sectores? ¿tales diferencias aluden al instrumento 
con que se miden los indicadores ODS-6? ¿la conciencia y la conducta orientada hacia la sustentabilidad es reflejo de estas diferencias?

A partir de estos interrogantes serán prioritarias líneas de investigación concernientes a la fiabilidad y validez de instrumentos que miden la diseminación de las ODS-6 en diferentes sectores y escenarios, así como la evidencia empírica de diferencias antes y después de talleres orientados hacia la sustentabilidad de los recursos y servicios hídricos municipales como residenciales.

A partir de estas indagatorias será preciso observar las diferencias y similitudes de la asimilación de los ODS-6 en instituciones públicas como en organizaciones privadas con la finalidad de anticipar las problemáticas endémicas de escasez, desabastecimiento, insalubridad y carestía.

Secuencias pedagógicas relativas a la orientación hacia la sustentabilidad en el aula, así como fuera de ella permitirán reducir el impacto de las problemáticas hídricas y maximizar los ODS-6 en la formación académica, profesional y laboral de las partes involucradas como los docentes y estudiantes.

A ese tenor, el diseño de prácticas docentes, evaluación del desempeño, planeación didáctica y manejo de contenidos deberán estar configurados desde la orientación hacia la sustentabilidad, incorporando cada uno de los ocho factores establecidos en el presente trabajo.

\section{Referencias}

Ato, M., López, J., y Benavente, A. (2013). Un sistema de clasificación de los diseños en investigación en psicología. Anales de Psicología, 23(3), 1038-1059. https://www.redalyc.org/pdf/167/16728244043.pdf.

Colmenarejo, R. (2016). El agua ¿Derecho humano? Revista de Fomento Social, 71(1), 99-102. file:///C:/Users/UNAM/Downloads/Dialnet-Agua-5609247.pdf.

Corral, V., Tapia, C., Fraijo, B., Mireles, J., y Márquez, P. (2008). Orientación hacia la sustentabilidad como determinante de los estilos de vida sustentables: Un estudio con una muestra mexicana. Revista Mexicana de Psicología, 5(2), 313327. https://www.redalyc.org/pdf/2430/243016308011.pdf.

Ferrando, P. J., y Anguiano, C. (2010). El análisis factorial como técnica de investigación psicológica. Papeles del Psicólogo, 3(1), 18-33. https://www.redalyc.org/articulo.oa?id=77812441003.

Gain, A., Giupponi, C., y Wada, Y. (2016). Measuring global water security towards sustainable development goals. Environmental Research Letters, 11(1), 1-14. https://iopscience.iop.org/article/10.1088/1748-9326/11/12/124015.

Gamboa, G. (2015). Los objetivos del desarrollo sostenible. Una propuesta bioética. $\begin{array}{llll}\text { Persona } \quad y & \text { Bioética, } & 19(2), & 175-181 .\end{array}$ https://www.redalyc.org/pdf/832/83242580001.pdf.

Kumar, B. (2014). Environmental education: The tool for sustainable development. Global Journal Multidisciplinary Studies, 3(10), 124-135.

Little, D. (2014). Defining sustainability in meaningful ways for education. Journal of Sustainability Education, 7, 1-19. 
Maletic, M., Maletic, D., Dahlgaard, J. J., Dahlgaard-Park, S., y Gomiscek, B. (2016). Effect of sustainability-oriented innovation practices on the overall organizational performance: an empirical examination, Total Quality Management and Business Excellence, 27 (9-10), 1171-1190. https://core.ac.uk/download/pdf/192594679.pdf.

Manzano, A. P. (2017). Introducción a los modelos de ecuaciones estructurales. Investigación en Educación Médica, 7(25), 67-72. http://www.scielo.org.mx/pdf/iem/v7n25/2007-5057-iem-7-25-67.pdf.

Merino, C. (2016). Diferencias entre alfas de Cronbach, con muestras y partes pequeñas. Un programa VB. Anales de Psicología, 32(2), 587-588. https://www.redalyc.org/articulo.oa?id=16745250031.

Milana, M., Rasmussen, P., y Holford, J. (2014). Public policy and sustainability of adult education. Encyclopaideia, XVIII(40), 3-13.

Noga, J., y Wolbring, G. (2012). The economic and social benefits and the barriers of providing people with disabilities accessible clean water and sanitation. Sustainability, 4, 3023-3041. DOI: 10.3390/su4113023.

Sanahuja, J. A., y Tezanos, S. (2017). Del milenio a la sostenibilidad: retos y perspectivas de la agenda 2030 para el desarrollo sostenible. Política y Sociedad, 50(2), 533-555. http://dx.doi.org/10.5209/POSO.51926.

Valizadeh, N., Bijani., y Hayati, D. (2019). A comparative analysis of behavior theories towards farmers' water conservation. International Journal of Agricultural Management and Development, 9(1), 1-10. http://ijamad.iaurasht.ac.ir/article_545944_4de15e816f5b7d0ec57de819a84d 21f9.pdf.

Yazici, 0. (2020). Awareness of hydrography courses students on protection freshwater resources. Review of International Geographical Education, 10(1), 97119. http://www.rigeo.org/vol10no1/Number1Spring/RIGEO-V10-N1-5.pdf. 